\title{
TITLE:
}

\section{(1)-D Shallow Water Models for Dam Break Flash Floods with Different Junction and Bend Treatments}

\section{$\operatorname{AUTHOR}(\mathrm{S})$ :}

Yoshioka, Hidekazu; Unami, Koichi; Fujihara, Masayuki

\section{CITATION:}

Yoshioka, Hidekazu ...[et al]. (1)-D Shallow Water Models for Dam Break Flash Floods with Different Junction and Bend Treatments. Communications in Computer and Information Science 2014, 474: 201-215

\section{ISSUE DATE:}

2014

URL:

http://hdl.handle.net/2433/199663

\section{RIGHT:}

The final publication is available at Springer via http://dx.doi.org/10.1007/978-3-66245289-9_18.; この論文は出版社版でありません。引用の際には出版社版をご確認ご利用 ください。; This is not the published version. Please cite only the published version. 


\title{
1-D Shallow Water Models for Dam Break Flash Floods with Different Junction and Bend Treatments
}

\author{
Hidekazu Yoshioka ${ }^{1}$, Koichi Unami ${ }^{2}$, and Masayuki Fujihara ${ }^{3}$ \\ ${ }^{1}$ Graduate School of Agriculture, Kyoto-University, Kyoto, Japan \\ yoshih@kais.kyoto-u.ac.jp \\ ${ }^{2}$ Graduate School of Agriculture, Kyoto-University, Kyoto, Japan \\ unamiladm.kais.kyoto-u.ac.jp \\ ${ }^{3}$ Graduate School of Agriculture, Kyoto-University, Kyoto, Japan \\ fujihara@kais.kyoto-u.ac.jp
}

\begin{abstract}
Recently-developed Finite Element/Volume Methods for the crosssectionally averaged 1-D shallow water equations in open channel networks are applied to idealized and real cases of dam break flash floods for their examination on robustness and versatility. The major differences among the schemes are the treatments of junctions and bends in the momentum equation as the internal boundary conditions. The computational results show robustness of the schemes. The results also show critical influences of the internal boundary conditions on the water surface profiles and flood arrival times, indicating that a necessary ingredient for their successful prediction is evaluation of the momentum flux with appropriately considering the channel configurations.
\end{abstract}

Keywords: Shallow water equations dam break flash flood Finite Element/Volume Method $(\mathrm{FEVM}) \cdot$ internal boundary condition.

\section{$1 \quad$ Introduction}

Dams have played major roles in basic human activities, such as irrigation and hydroelectric generation. However, dams always associate failure risks resulting in flash floods potentially involving the loss of human life. Singh [1] summarizes more than 60 dam failures over the world, which include the St. Francis Dam failure in the United States of America in 1928 [2] and the Malpasset Dam failure in France in 1959 [3], both of which resulted in more than several hundred deaths. Failures of earthen dams associated with irrigation tanks in Japan have been documented in Uchida [4]. Failures of natural dams, such as landslide dams [5] and glacial-ice dams [6], have also been reported in the literatures. Costa and Schuster [7] summarize formation and failure mechanisms of natural dams. Prediction and risk analysis for dam break flash floods are key research topics in civil and environmental engineering because of their catastrophic impacts on human life and activity. Numerical simulation based on the shallow water equations (SWEs) [8], which is a nonlinear hyperbolic system of partial differential equations with source terms, in particular have been serving as an effective tool for these purposes. The SWEs are broadly categorized into the following two 
types: the cross-sectionally averaged 1-D SWEs and the depth-averaged horizontally 2-D ones. Various numerical schemes have been developed for solving the SWEs, most of which rely on the finite volume method (FVM) based on the Riemann-solvers with the high-resolution techniques [9]. A weakness of these numerical schemes is the complexity in spatial discretization that may result in significant loss of computational efficiency. On the other hand, some researchers developed simpler and sufficiently accurate numerical schemes that rely neither on the Riemann-solvers nor on the highresolution techniques [10-12]. The authors developed staggered numerical schemes for the 1-D and 2-D SWEs with heuristic upwind methods for the momentum flux and the source terms based on the local Froude numbers [13-15].

For simulating dam break flash floods in mountainous areas where the watercourses are narrow and steep in particular, the 1-D SWEs work more effectively than the 2D counterparts [16]. However, numerical resolution of the 1-D SWEs in such a case encounters a number of difficulties, such as irregular cross-sectional shape, channel bend, channel junction, wet and dry interface, shock wave, and depression wave. An accurate and versatile numerical scheme is necessary to solve the 1-D SWEs under the above-mentioned severe computational conditions. Although various numerical schemes for the 1-D SWEs have been developed in the literatures, only a few of them have been applied to real problems of dam break flash floods in mountainous areas mainly due to the difficulty in handling the above-mentioned issues.

The purpose of this paper is to apply different numerical schemes to dam break flash floods in idealized and real cases for their examination on robustness and versatility. The schemes examined are the Finite Element/Volume Method (FEVM) of Ishida et al. [17], that of Unami and Alam [13], and that of Yoshioka et al. [15]. Major differences among them are treatments of the junctions and bends as the internal boundary conditions (IBCs) whose influences are significant for the flows in the mountainous areas where both of the hydraulic elements are commonly encountered.

The remainder of this paper is organized as follows. The 1-D SWEs are presented in section 2. The IBCs at the junctions and bends for the numerical schemes are presented in section 3. Section 4 performs applications of the schemes to the idealized and real cases of dam break flash floods. Section 5 provides conclusions of this paper.

\section{Shallow Water Equations}

The 1-D SWEs govern surface water flows based on cross-sectionally averaged variables. The 1-D SWEs defined along a single channel consist of the continuity equation

$$
\frac{\partial A(\eta)}{\partial t}+\frac{\partial Q}{\partial s}-q=0
$$

and the momentum equation

$$
\frac{\partial Q}{\partial t}+\frac{\partial F}{\partial s}+g A\left(\frac{\partial \eta}{\partial s}+S_{\mathrm{f}}\right)=0
$$


with the momentum flux $F$ given by

$$
F=\frac{\beta Q^{2}}{A}
$$

where $t$ is the time, $s$ is the 1-D abscissa taken along the channel, $A$ is the crosssectional area of flow, $\eta$ is the water surface elevation, $Q$ is the discharge, $q$ is the lateral inflow, $g$ is the gravitational acceleration, $\beta \geq 1$ is the momentum correction coefficient, and $S_{\mathrm{f}}$ is the 1-D Manning's friction slope given by

$$
S_{\mathrm{f}}=\frac{n^{2} Q|Q|}{A^{2} R^{4 / 3}}
$$

where $n$ is the Manning's coefficient and $R$ is the hydraulic radius. For the flows in open channel networks, it is reasonable to rewrite (1) in the integral form

$$
\int_{\Omega \cap B(y, r)} T \frac{\partial \eta}{\partial t} \mathrm{~d} s+\sum_{j=1}^{v} \sigma_{j} Q_{r, j}+\sum Q_{\mathrm{B}}=\int_{\Omega \cap B(y, r)} q \mathrm{~d} s
$$

where $\Omega$ represents the domain of the flow as a locally 1-D open-channel network [15], $T=\frac{\partial A}{\partial \eta}$ is the top width of the water surface, $B(y, r)$ is the horizontally 2-D $r$ -neighborhood of a point $y \in \Omega$ with sufficiently small $r>0, v$ is the total number of reaches that intersect with the boundary of $B(y, r), Q_{r, j}$ is the discharge at the $j$ th intersection, $\sum Q_{\mathrm{B}}$ represents the sum of the discharges specified at the boundary vertices in $\Omega \bigcap B(y, r)$, and $\sigma_{j}$ is the sign parameter defining the direction of the abscissa in the $j$ th reach. Here, $\sigma_{j}$ is equal to 1 when the $x$ abscissa in the $j$ th reach is facing outward to the boundary of $B(y, r)$ and is otherwise equal to -1 . Application of the integral-based continuity equation (5) to single channels recovers the conventional one (1). The continuity equation serves as an IBC at the junctions. In fact, taking the limit $r \rightarrow+0$ in (5) yields the local mass conservation law

$$
\sum_{j=1}^{v} \sigma_{j} Q_{r, j}=\int_{\Omega \bigcap B(y, r)} q \mathrm{~d} s
$$

The momentum equation (2) must also be equipped with an IBC at a junction or at a bend. Without the loss of generality, a junction connecting $m$ inflow reaches and $n$ outflow reaches is considered since the IBC is local in nature. A junction with $m=n=1$ is regarded as a bend. The $s$ abscissae along the inflow reaches end at the junction and those along the outflow reaches start from the junction. The momentum 
flux $F$ at the downstream-end of the $i$ th inflow reach is denoted by $F_{i, \mathrm{ds}}$ and that at the upstream-end of the $j$ th outflow reach by $F_{j \text {,us }}$. The momentum fluxes $F_{i, \text { ds }}$ and are $F_{j \text {,us }}$ non-negative by the definition. Assuming a linear dependence of $F_{j \text {,us }}$ on $F_{i, \text { ds }}$ leads to the IBC for the momentum equation as

$$
F_{j, \mathrm{us}}=\sum_{i=1}^{m} \mu_{i, j} F_{i, \mathrm{ds}}
$$

where $\mu_{i, j}$ is the non-negative coefficient to be determined.

\section{$3 \quad$ Numerical Schemes}

The 1-D SWEs are solved with one of the three numerical schemes, which are the FEVM of Ishida et al. [17], that of Unami and Alam [13], and that of Yoshioka et al. [15]. The three numerical schemes are referred to as M1-scheme, M2-scheme, and M3-scheme, respectively. Basically, these numerical schemes share a common spatial discretization procedure except for the IBC of the momentum equation. The temporal term of the continuity equation is fully lumped to enhance stability in handling wet and dry interfaces. The IBC (7) for each numerical scheme is specified as follows.

- The FEVM of Ishida et al. [17] (M1-scheme)

$$
F_{j, \text { us }}=\sum_{i=1}^{m} F_{i, \mathrm{ds}} \quad\left(\mu_{i, j}=1\right)
$$

- The FEVM of Unami et al. [13] (M2-scheme)

$$
F_{j, \text { us }}=\sum_{i=1}^{m} \max \left(\cos \theta_{i, j}, 0\right) F_{i, \mathrm{ds}} \quad\left(\mu_{i, j}=\max \left(\cos \theta_{i, j}, 0\right)\right)
$$

where $0 \leq \theta_{i, j} \leq 2 \pi$ is the crossing angle between the $i$ th inflow reach and the $j$ th outflow reach.

- The FEVM of Yoshioka et al. [15] (M3-scheme)

$$
F_{j, \text { us }}=\lambda_{j} \sum_{i=1}^{m} \max \left(\cos \theta_{i, j}, 0\right) F_{i, \mathrm{ds}} \quad\left(\mu_{i, j}=\lambda_{j} \max \left(\cos \theta_{i, j}, 0\right)\right)
$$

with the non-negative coefficient $\lambda_{j}$ given by 


$$
\lambda_{j}=\frac{Q_{j}}{\sum_{j=1}^{n} Q_{j}},
$$

which is replaced by 0 if its denominator vanishes. In the M1-scheme, the momentum equation (2) is regarded as the governing equation of the discharge $Q$ as a scalar variable, while it is regarded as a vector variable in the M2-scheme and M3-scheme. This difference of the physical consideration among the schemes results in the difference in their formulations, which is indicated by the inclusions of the coefficient $\cos \theta_{i, j}$ in the IBCs (9) and (10). The qualitative difference among the IBCs (8) through (10) are characterized with the momentum difference $\Delta F$ defined by

$$
\Delta F=\sum_{j=1}^{n} F_{j, \text { us }}-\sum_{i=1}^{m} F_{i, \mathrm{ds}} .
$$

From the physical point of view, the momentum difference $\Delta F$ should not increase at the junction since its increase means the existence of unphysical external forces at the point. According to Yoshioka et al. [15], the IBCs (8), (9), and (10) satisfy

$$
\Delta F \geq 0,
$$

$$
\Delta F \leq 0
$$

if the condition

$$
\sum_{i=m+1}^{m+n} \cos \theta_{i, j} \leq 1
$$

holds, and

$$
\Delta F \leq 0,
$$

respectively, showing that only the M3-scheme unconditionally complies with the non-increase of $\Delta F$. The M1-scheme does not comply with the condition, while the M2-scheme does if the channel configuration satisfies (15), which is satisfied for a bend. Influences of the IBCs on the steady flows in experimental open channels have been investigated in Yoshioka et al. [15], indicating advantages of the M3-scheme over the others in simulating the flows with accurate prediction of the discharge for the downstream reaches of a junction. It should be noted that no or at least only a few numerical schemes for the 1-D SWEs except for the M2-scheme and the M3-scheme consider momentum losses at the junctions and bends as the physically-based IBCs. 


\section{$4 \quad$ Numerical Simulation}

The numerical schemes presented in the previous section are applied to idealized and real cases of dam break flash floods to investigate their robustness and versatility. Since these numerical schemes have already been verified with the standard benchmark problems, such as the dam break problems in flat and friction-less channels and the steady flows over a bump $[13,15,17]$, this paper focuses on more complicated situations involving junctions and bends.

\subsection{Dam Break Problem in a Hypothetical Open Channel Network}

Numerical simulation of an idealized dam break problem in a hypothetical multiplyconnected open channel network is firstly performed. The computational domain considered is shown in Fig. 1, in which key nodes are alphabetically labelled from A through G: the upstream-end A, the downstream-end $\mathrm{E}$, the junctions $\mathrm{B}$ and $\mathrm{D}$, the bends $\mathrm{C}$ and $\mathrm{F}$, and the dam $\mathrm{G}$ located $50.0(\mathrm{~m})$ downstream of the upstream-end $\mathrm{A}$. The dam $G$ is assumed to be a vertical wall with an infinitesimal width. The length of the reaches A-B and D-F is set as $200.0(\mathrm{~m})$ and the others as $282.8(\mathrm{~m})$. Channel slope of the reaches A-B and D-F is set as 0.010 and those of the others as 0.007. The elevation at the downstream-end $\mathrm{E}$ is set as $0(\mathrm{~m})$. Cross-sectional shape of the channels is a rectangle with the width of $4(\mathrm{~m})$ in the reaches B-C-D, $10(\mathrm{~m})$ in the reach A-G, and $2(\mathrm{~m})$ in the other reaches. Manning's roughness coefficient is set as 0.03 $\left(\mathrm{s} / \mathrm{m}^{1 / 3}\right)$ over the computational domain. The upstream-end $\mathrm{A}$ and the downstream-end $\mathrm{E}$ are solid walls where the water cannot penetrate. The reach A-G serving as a reservoir is filled with a still water whose water surface elevation is equal to $20(\mathrm{~m})$. The reaches downstream of the dam $G$ are initially assumed to be dry where the water depth of $0.0001(\mathrm{~m})$ is artificially specified to prevent the division by 0 in the numerical schemes. The dam $\mathrm{G}$ is instantaneously and completely removed at the initial time $t=0(\mathrm{~s})$. In the present case, the crossing angles between the reaches A-B and B-C and that of between the reaches A-B and B-F equal to 45 (deg), indicating that the condition (15) is not satisfied since its left hand side equals to $\sqrt{2}>1$. The reaches are discretized into elements with the length of $0.5(\mathrm{~m})$. The computational domain is consequently discretized into 1,200 elements with 1,200 nodes. The time increment $\Delta t$ for the temporal integration in the numerical schemes is fixed to 0.005 (s).

Figs. 2 through 4 show computed water surface profiles with the M1-scheme, the M2-scheme, and the M3-scheme, respectively. All the numerical schemes compute the flows without numerical instability and significant spurious oscillations. The wet and dry interfaces are reasonably captured in the numerical solutions. There are qualitative differences among the numerical solutions around the bends $\mathrm{C}$ and $\mathrm{F}$ where the momentum in the flow should be dissipated due to the abrupt changes of the flow directions. Experimental investigations showed that an unsteady supercritical flow passing through a bend associates a bore propagating upstream [18], which cannot be reproduced with the M1-scheme because it does not consider the effect of the bend in its formulation. On the other hand, the M2-scheme and the M3-scheme reproduce the 
bores, qualitatively agreeing with the results observed in the similar hydraulic experiments [18]. Another major difference among the numerical schemes is the behaviour of the flow around the junction B as shown in Figs. 2 through 4 and an enlarged view Fig. 5. The flows with the M1-scheme and the M2-scheme involve convex water surface profiles in the upstream area of the reach B-C (just downstream of the junction B), which is suppressed in the M3-scheme. Fig. 6 shows the computed local Froude numbers along the reaches A-G-B-C at the time $t=36(\mathrm{~s})$. The flows around the junction $\mathrm{B}$ at that time are supercritical for all the numerical schemes, attaining local maximum values of the Froude numbers just upstream of the junction. The magnitudes of the local maximum values are the largest in the M1-scheme and the smallest in the M3-scheme.

The flood arrival times at the downstream-end E with the M1-scheme, the M2scheme, and the M3-scheme are computed as 236.6 (s), 242.0 (s), and 249.2 (s), respectively. The difference in the flood arrival times is a consequence of the difference of the IBCs at the junctions and bends. The IBCs (8) through (10) indicate that the loss of the momentum in the flow is smallest in the M1-scheme and the largest in the M3-scheme, that of the M2-scheme lies between them. Since the larger momentum flux results in the larger acceleration of flow, the obtained results on the flood arrival times are consistent with the intuitive consideration. The computational results presented in this sub-section indicate critical influences of the IBCs on simulating the dam break flash flood with the 1-D SWEs.

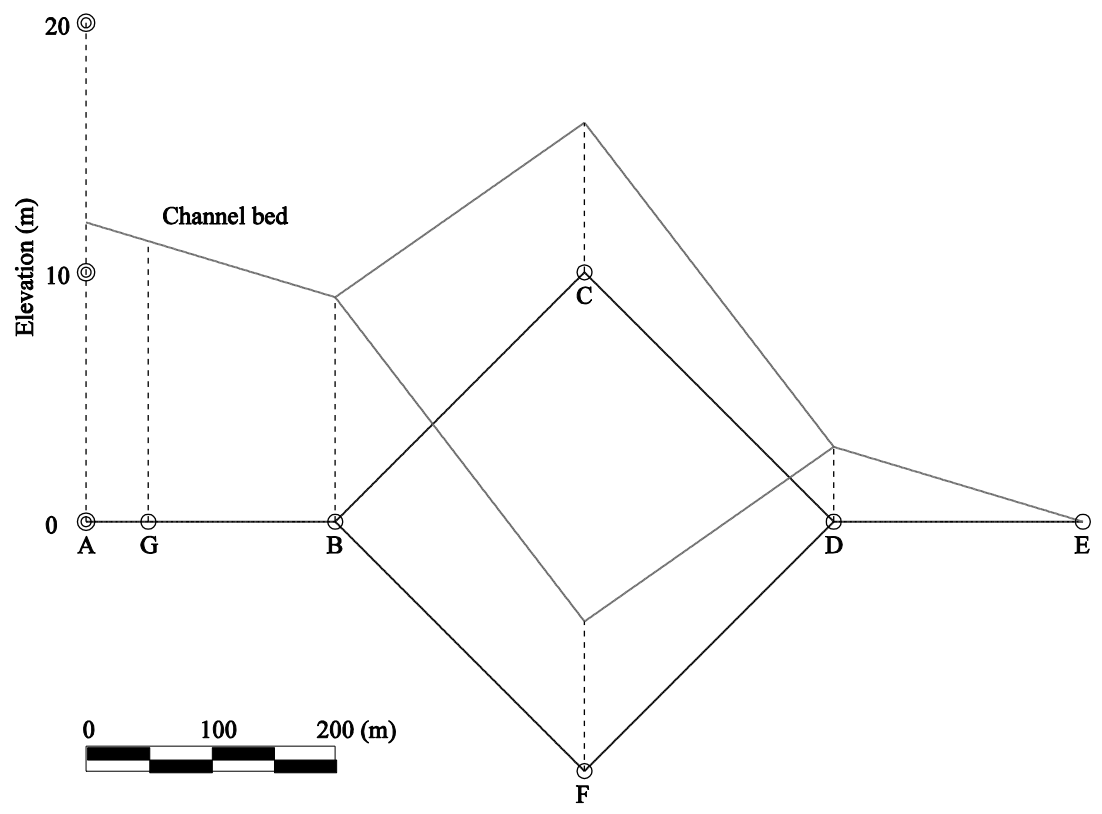

Fig. 1. Computational domain for the idealized dam break problem 


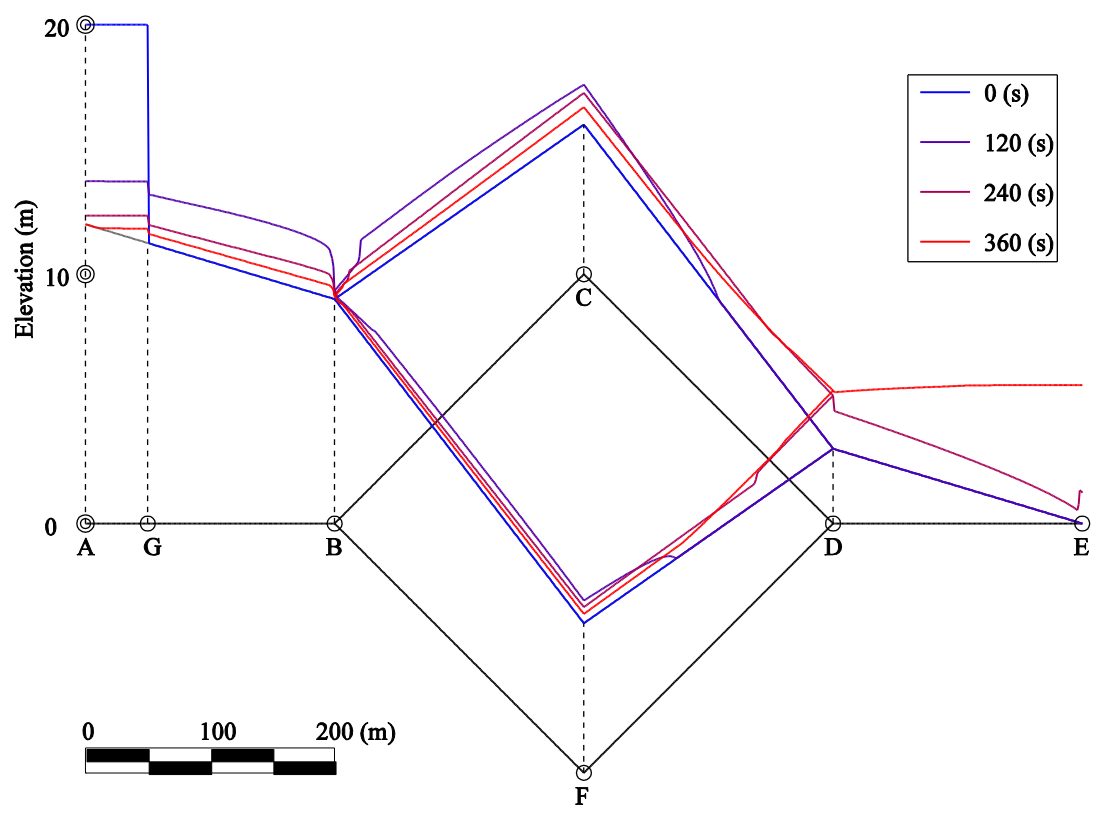

Fig. 2. Computed water surface profiles with the M1-scheme

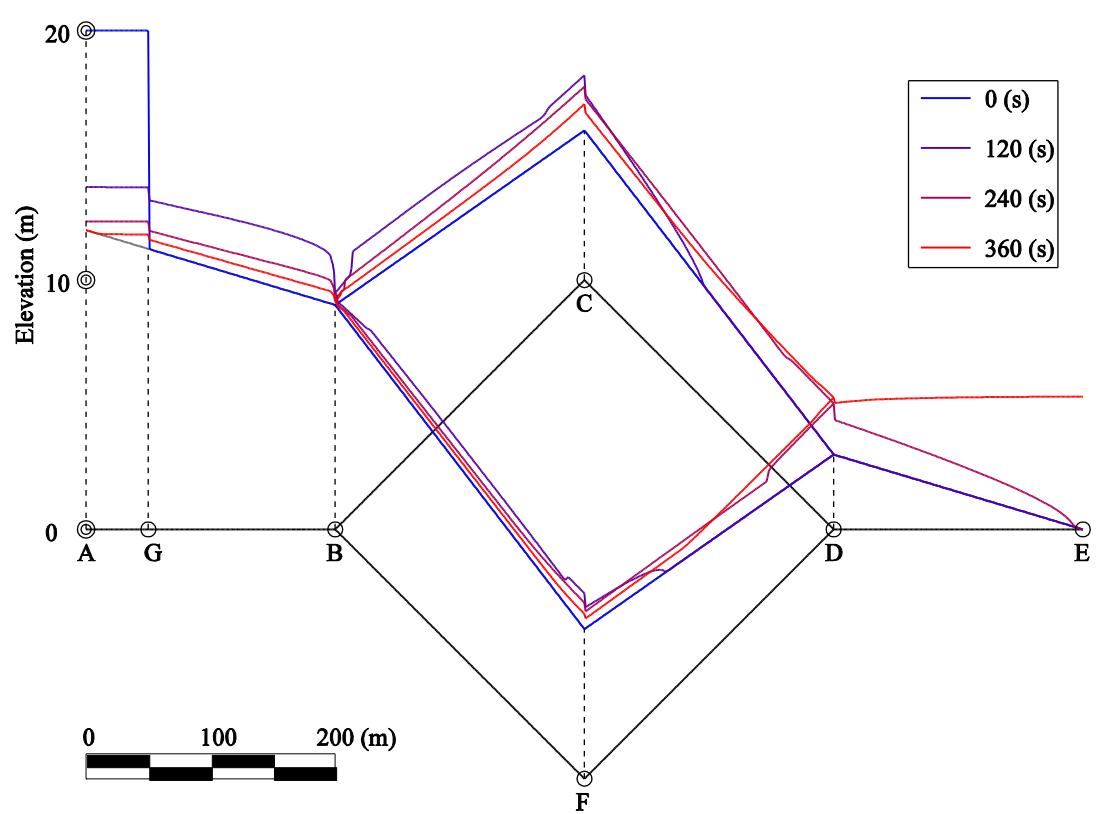

Fig. 3. Computed water surface profiles with the M2-scheme 


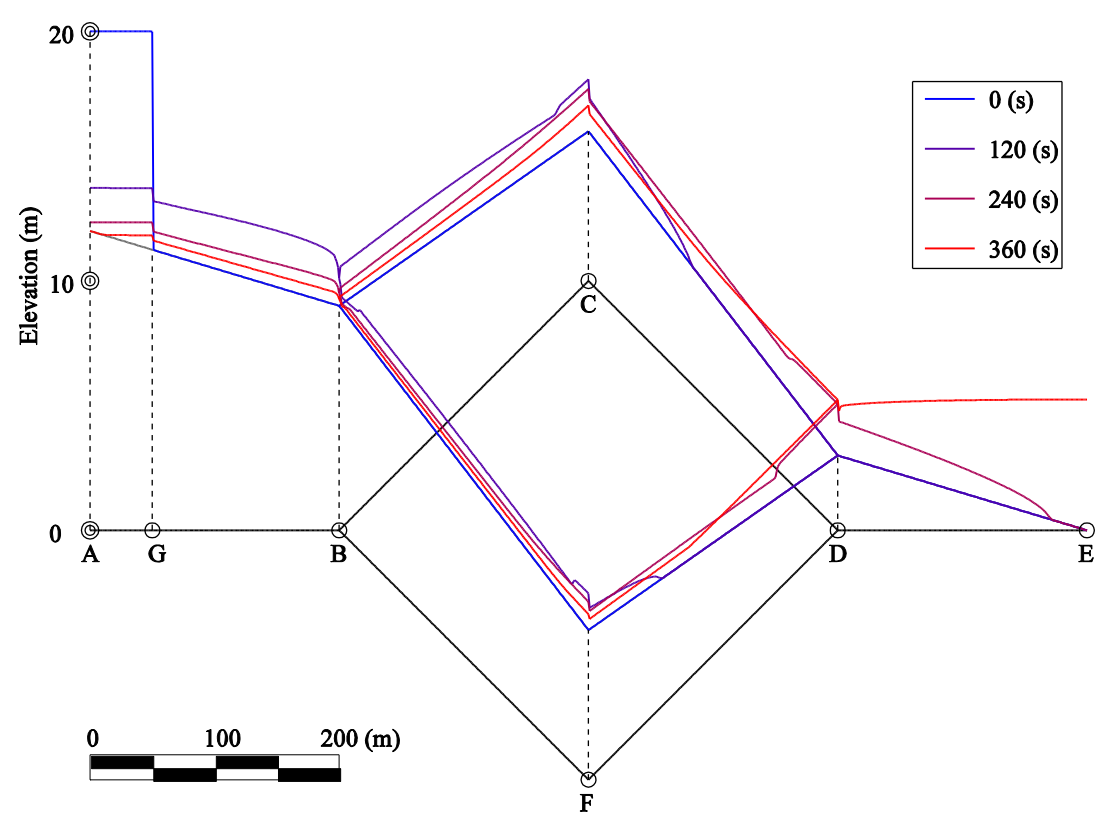

Fig. 4. Computed water surface profiles with the M3-scheme

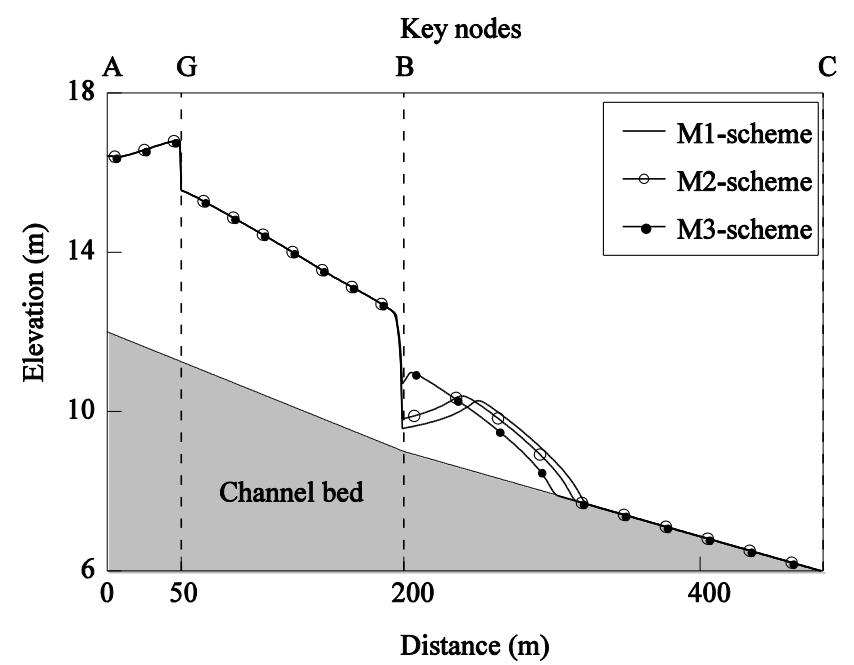

Fig. 5. Computed water surface profiles along the reaches A-G-B-C at $t=36$ (s) 


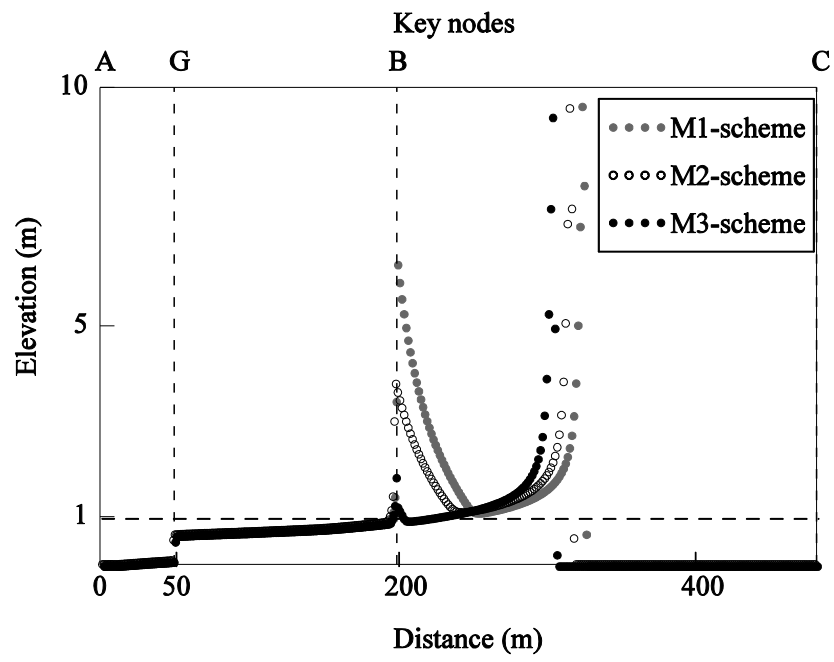

Fig. 6. Computed Froude numbers along the reaches A-G-B-C at $t=36$

$6(s)$

\subsection{Earthquake-induced Dam Break Flash Flood in Japan}

The three numerical schemes are applied to a dam break flash flood due to a huge earthquake. The study area is Fujinuma reservoir (Sukagawa City in Fukushima Prefecture, Japan) and its downstream area where the villages and farmlands existed as shown in Fig. 7. The reservoir associated Fujinuma Dam (N37.302, E140.195; WGS 84), an earth-fill type dam whose main embankment completely failed on March 11 in 2011 due to the Great East Japanese Earthquake with the maximum magnitude of 9.0 [19]. Distance between the dam and the epicenter of the earthquake was approximately $240 \mathrm{~km}$. The failure of the main embankment of Fujinuma Dam resulted in a flash flood, which hit Taki village at its downstream within several minutes from the failure, washing away 19 houses, flooding 155 houses below floor level, and killing eight people [20]. The main embankment is currently in reconstruction. The auxiliary embankment of Fujinuma Dam, although which did not fail, was significantly damaged by the earthquake [19]. This fact indicates that there were potential risks of simultaneous failures of the main and auxiliary embankments.

Numerical simulation in this sub-section focuses on an application of the presented schemes to a dam break flash flood due to simultaneous failures of the main and auxiliary embankments of Fujinuma Dam, which can be a possible scenario because both of the embankments were significantly damaged due to the earthquake. The computational domain is a multiply connected locally 1-D open channel network containing the reservoir and its downstream channels as shown in Fig. 8. The computational domain consists of 327 elements and 327 nodes [21]. The domain has a number of bends whose modelling plays a crucial role in the present numerical simulation as shown in later. The cross-sectional shape of the channels is determined from the 
available topographical data in Google Map (Google Inc.). The upstream-end of the domain (A) is an impermeable wall. There are four junctions in the computational domain, which are the vertices B, D, I, and E, one of which (B) is in the reservoir serving as a diverging point of the flow. The vertices $C$ and $G$ are the main and auxiliary embankments. The vertex $\mathrm{F}$ is the downstream-end of the domain where a hydraulic drop is installed. The Manning's roughness coefficient is set as $0.035\left(\mathrm{~s} / \mathrm{m}^{1 / 3}\right)$ and the momentum correction coefficient as 1.1. Initial condition for the simulation is a steady flow with the constant discharges of $10\left(\mathrm{~m}^{3} / \mathrm{s}\right)$ at the upstream-ends $\mathrm{J}$ and $\mathrm{K}$ and the critical flow condition at the downstream-end F. Initially the reservoir is assumed to be at the full capacity with the maximum water depth of $18.5(\mathrm{~m})$. Both of the embankments are instantaneously removed at the initial time $t=0$ (s).

Fig. 9 shows the computed water depth time series at the vertices D (Taki Village), $\mathrm{F}$ (downstream-end), and $\mathrm{H}$ (downstream-side of the southern valley) with the three numerical schemes. The computed water depths with the M2-scheme and the M3scheme are not-distinguishable in the figure, while that with the M1-scheme is clearly different from the others around the peaks in particular. This difference results from the physically invalid formulation of the M1-scheme that does not consider the momentum losses due to the existence of the junctions and bends. The computed results at the vertex D, at which the flash flood from the main embankment directly strikes, shows that the peak water depth with the M1-scheme is lower than $90 \%$ of those with the others. The M1-scheme also underestimates the water depth at the vertex H. The computed results at the vertex F (downstream-end of the domain) shows that the M1scheme predicts 130 (s) earlier flood arrival time than the others.

Fig. 10 shows the computed hazard maps, indicating that there is no significant difference among the inundation maps with the three numerical schemes. The total inundated areas are computed as $3.651\left(\mathrm{~km}^{2}\right), 3.537\left(\mathrm{~km}^{2}\right)$, and $3.536\left(\mathrm{~km}^{2}\right)$ with the M1scheme, the M2-scheme, and the M3-scheme, respectively; relative difference among them are smaller than $4(\%)$. This is considered due to the fact that the hazard maps do not reflect dynamic behaviour of the flash flood. The hazard maps indicate severely flooded areas around the vertex D and the vertex I (Paddy field).

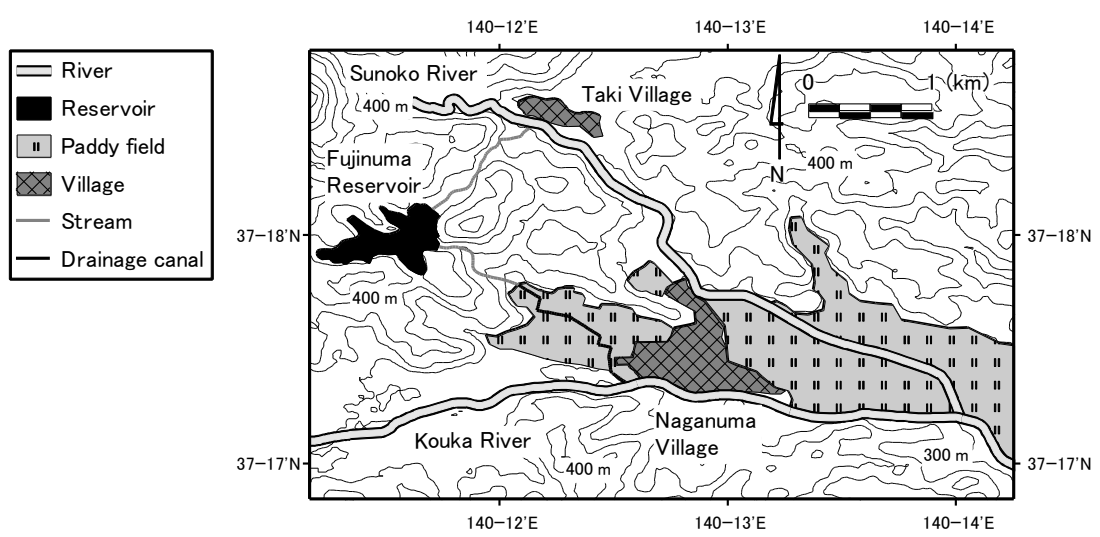

Fig. 7. Map of the study area 


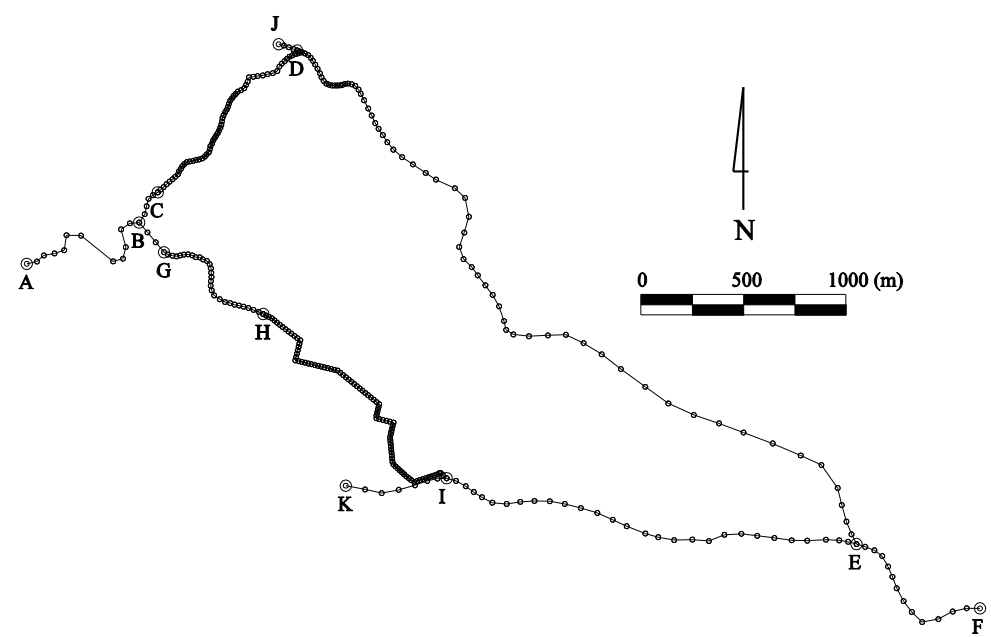

Fig. 8. Sketch of the computational mesh with key nodes

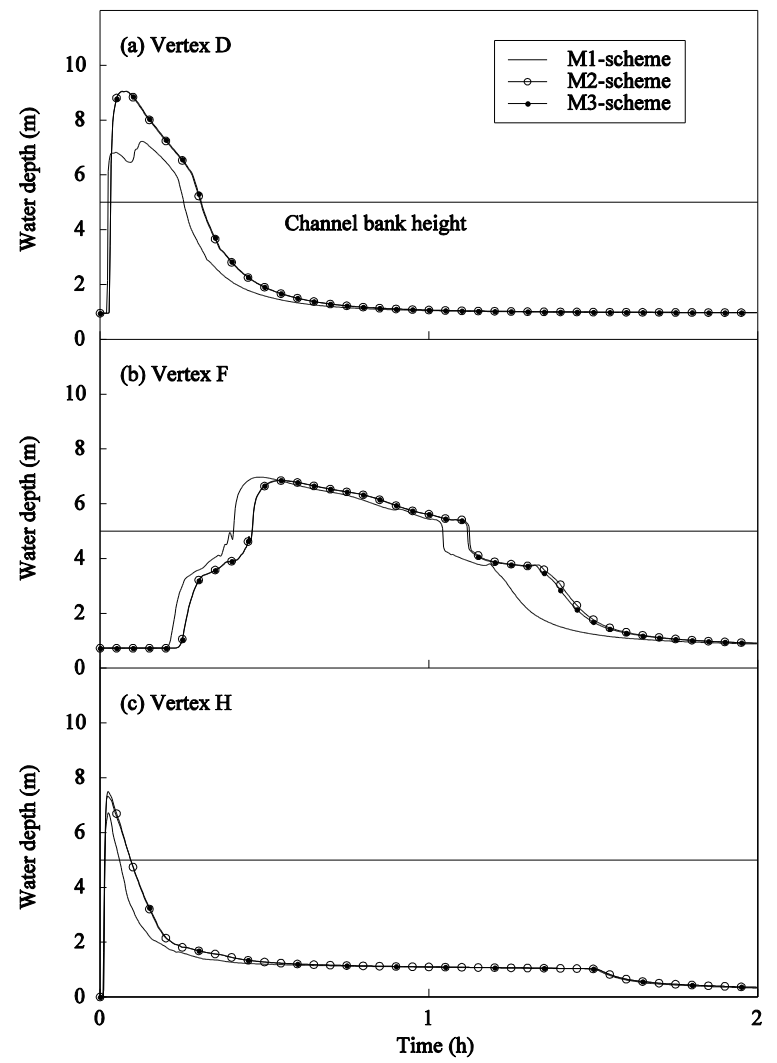

Fig. 9. The water depth time series at the vertices D, F, and H 

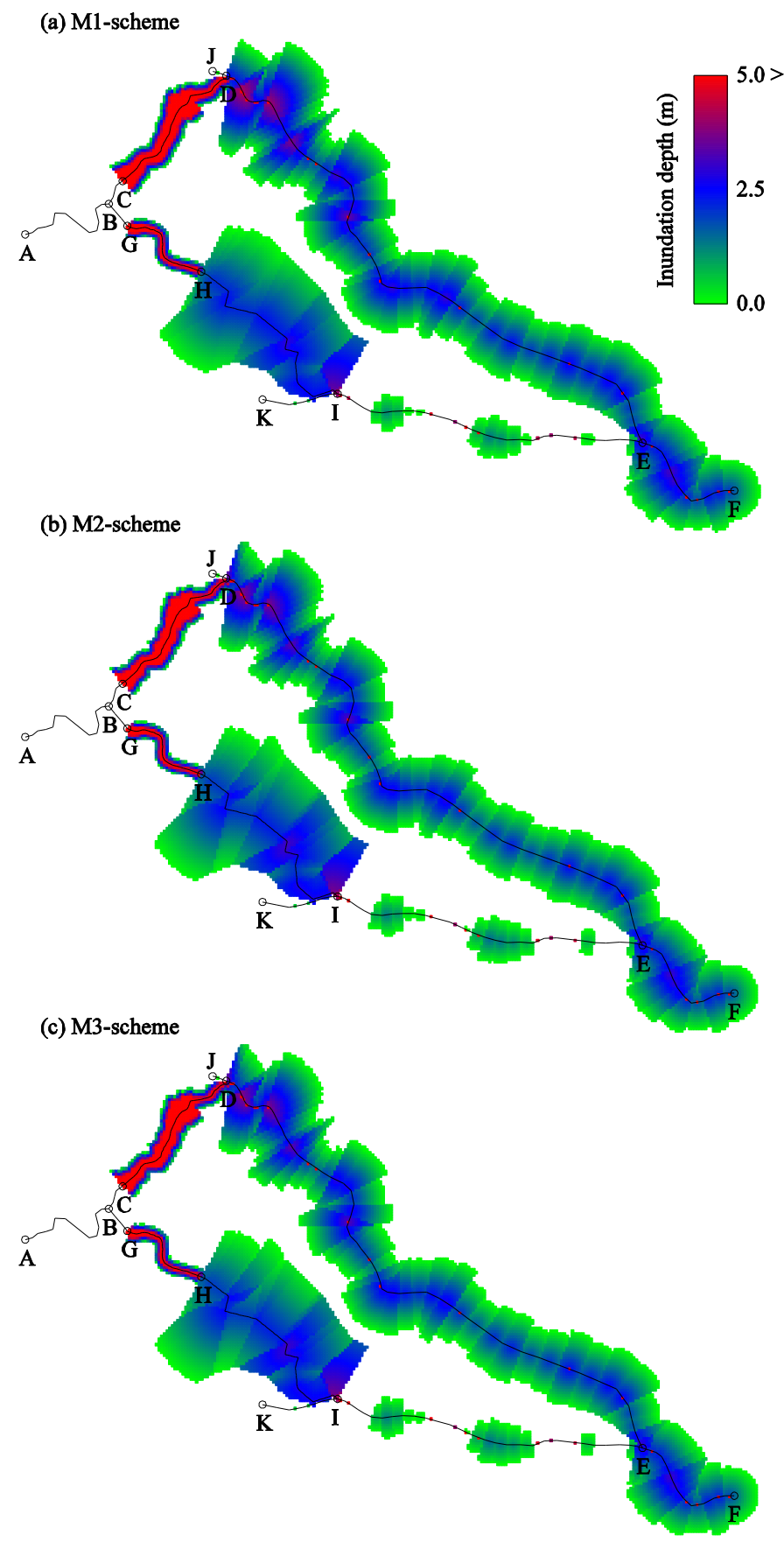

Fig. 10. Hazard maps with the three numerical schemes 


\section{Conclusions}

The three numerical schemes for solving the 1-D SWEs, which are the M1-scheme, the M2-scheme, and the M3-scheme, were presented and applied to dam break flash floods in the idealized and real cases. All the numerical schemes computed the solutions without numerical instability and significant spurious oscillations. The computational results revealed qualitative differences among the numerical schemes in simulating the flash floods. The computational results of the idealized test case showed that the M1-scheme cannot simulate the bores propagating upstream from bends that the other schemes can reasonably simulate. This difference stemmed from the difference in their formulations; whether the momentum flux is treated as a vector variable or not. Quantitative difference among the numerical schemes was also indicated in the numerical simulation of the dam break flash flood due to the simultaneous failures of the main and auxiliary embankments of Fujinuma Dam in Fukushima Prefecture, Japan. The flood arrival times at the key nodes were remarkably different among the numerical schemes, implying the importance of the IBC in applications. The computed hazard maps were not clearly different among the numerical schemes. Future research will focus on implementations of the numerical schemes to risk analysis on flash floods due to failures of dams in Japan whose risk analysis have not been carried out yet regardless of their high potential failure risks.

\section{Acknowledgement}

This research was supported by JSPS research grant No. $25 \cdot 2731$.

\section{References}

1. Singh, V.P.: Dam Breach Modeling Technology, pp. 6-100, Kluwer Academic Publications (1996)

2. Begnudelli, L., Sanders, B.F.: Simulation of the St. Francis dam-break flood. J. Eng. Mech. 133(11), 1200-1212 (2007)

3. Valiani, A., Caleffi, V., Zanni, A.: Case study: Malpasset dam-break simulation using a two-dimensional finite volume method. J. Hydraul. Eng. 128(5), 460-472 (2002)

4. Uchida, K.: Disasters on Irrigation Ponds and Conservation of Regional Environment in Japan, pp. 103-129, Kaisei-sha Publishing (2003) (in Japanese)

5. Dai, F.C., Lee, C.F., Deng, J.H., Tham, L.G.: The 1786 earthquake-triggered landslide dam and subsequent dam-break flood on the Dadu River, southwestern China. Geomorphology. 65(3-4), 205-221 (2005)

6. Shrestha, B.B., Nakagawa, H.: Assessment of potential outburst floods from the Tsho Rolpa glacial lake in Nepal, Nat. Hazards, 71(1), 913-936 (2014)

7. Costa, J.E., Schuster, R.L.: The formation and failure of natural dams. Geol. Soc. Am. bull. 100, 1054-1068 (1988)

8. Cunge, J.A., Holly Jr., F.M., Verwey, A.: Practical Aspects of Computational River Hydraulics, pp. 7-52, Pitman Publishing (1980)

9. Toro, E.F., Garcia-Navarro, P.: Godunov-type methods for free-surface shallow water flows: A review, J. Hydraul. Res. 45(6), 736-751 (2007) 
10. Capart, H., Eldho, T.I., Huang, S.Y., Young, D.L., Zech, Y.: Treatment of natural geometry in finite volume river flow computations. J. Hydraul. Eng. 129(5), 385-393 (2002)

11. Catella, M., Paris, E., Solari, L.: Conservative scheme for numerical modeling of flow in natural geometry. J. Hydraul. Eng. 134(6), 736-748 (2008)

12. Ying, X., Khan, A.A., Wang, S.S.Y.: Upwind conservative schemes for the Saint Venant equations. J. Hydraul. Eng. 130(10), 977-987 (2004)

13. Unami, K., Alam, A.H.M.B.: Concurrent use of finite element and finite volume methods for shallow water equations in locally 1-D channel networks. Int. J. Numer. Methods Fluids. 69(2), 255-272 (2012)

14. Yoshioka, H., Unami, K., Fujihara, M.: A finite element/volume method model of the depth averaged horizontally 2-D shallow water equations. Int. J. Numer. Methods Fluids. 75(1), 23-45 (2014)

15. Yoshioka, H., Unami, K., Fujihara, M.: Comparative numerical analysis on momentum flux evaluation schemes for shallow water flows in open channel networks. Journal of. Rainwater Catchment Systems. 19(2), 25-33 (2014)

16. Petaccia, G., Natale, E., Velickovic, M., Zech, Y., Soares-Frazão, S.: Flood wave propagation in steep mountainous rivers. J. Hydroinform. 120-137 (2013)

17. Ishida, K., Yangyuoru, M., Unami, K., Kawachi, T.: Application of shallow water equations to analyze runoff processes in hilly farmlands. Paddy Water Environ. 9(4), 393-401 (2011)

18. Soares-Frazao, S., Zech, Y.: Dam break in channels with $90^{\circ}$ bend. J. Hydraul. Eng. 128(11), 156-968 (2011)

19. Harder Jr., L.F., Kelson, K.I., Kishida, T., Kayen, R.: Preliminary Observations of the Fujinuma Dam Failure Following the March 11, 2011 Tohoku Offshore Earthquake, Japan. Geotechnical Extreme Events Reconnaissance Report No. GEER-25.e 2011, pp. 1-29 (2011)

20. Kahoku Online Network. http://www.kahoku.co.jp/spe/spe_sys1072/20110518_01.htm. Last Accessed on September 17, 2012. (in Japanese)

21. Yoshioka, H., Unami, K., and Fujihara, M. (2013) A dual grid based finite volume model for dam break flows in multiply connected open channel networks with general crosssections. Proceedings of the 62nd National Congress of Theoretical and Applied Mechanics, Tokyo, Japan, OS19-09, 1-2. (in Japanese) 